\title{
Sistema Renina Angiotensina Aldosterona em mulheres que utilizam contraceptivo hormonal injetável: protocolo de um estudo observacional comparativo de corte transversal
}

\author{
Renin Angiotensin Aldosterone System in women \\ using injectable hormonal contraceptive: protocol of a \\ comparative observational study of cross-sectional
}

\section{Priscilla Araújo dos Santos ${ }^{1}$ (i) Alice Miranda de Oliveira ${ }^{2}$ (1) Jefferson Petto ${ }^{3}$ (1)}

${ }^{1}$ Autora para correspondência. Escola Bahiana de Medicina e Saúde Pública (Salvador). Bahia, Brasil. priscilaaraujo10@hotmail.com

${ }^{2-3}$ Centro Universitário Social da Bahia (Salvador). Bahia, Brasil. alicemofisio@gmail.com, gfpecba@bol.com.br

\begin{abstract}
RESUMO | INTRODUÇÃO: Os contraceptivos orais são a forma mais utilizada para o controle de natalidade, chegando a 200 milhões de usuárias desde sua iniciação na década de 1960. Desde 2013, nosso grupo de pesquisa tem apresentado resultados que sugerem que mulheres em uso de Contraceptivos Orais Combinados (COC), e sem outros fatores de risco, apresentam maior valor de proteína $\mathrm{C}$ reativa, lipemia pós-prandial, lipoproteína de baixa densidade oxidada e diminuição da sensibilidade insulínica, quando comparadas a suas congêneres sem uso de COC. Recentemente, foi verificado que o uso de COC eleva os valores de renina plasmática em $600 \%$, podendo explicar por que o uso desse fármaco é um fator de risco para o desenvolvimento de hipertensão arterial sistêmica. Apesar de o uso de Contraceptivo Hormonal Injetável (CHI) estar aumentando, não encontramos estudos clínicos que abordassem o tema, demonstrando uma lacuna na literatura científica. OBJETIVO: Comparar os valores de renina plasmática, enzima conversora de angiotensina 1 e aldosterona de mulheres que utilizam $\mathrm{CHI}$ com mulheres que não utilizam nenhum contraceptivo à base de hormônio. MÉTODOS: Protocolo de um estudo observacional comparativo de corte transversal, composto por mulheres com idade entre 18 e 30 anos, eutróficas, irregularmente ativas pelo Questionário Internacional de Atividade Física, versão curta, que estão em uso continuado de $\mathrm{CHI}$ há pelo menos 6 meses ou que não fazem uso. A amostra será por conveniência, as participantes selecionadas assinarão o termo de consentimento livre e esclarecido. Posteriormente, responderão a um questionário padrão, serão submetidas a um exame físico, e serão encaminhadas para coleta das amostras sanguíneas.
\end{abstract}

PALAVRAS-CHAVE: Anticoncepcionais. Sistema ReninaAngiotensina. Hipertensão. Saúde da Mulher.
ABSTRACT | INTRODUCTION: Oral contraceptives are the most widely used form of birth control, reaching 200 million users since its inception in the 1960. Since 2013, our research group has presented results that suggest that women using Combined Oral Contraceptives (COC) and without other risk factors, have a higher value of C-reactive protein, postprandial lipemia, oxidized low-density lipoprotein and decreased insulin sensitivity, when compared to their counterparts without the use of COC. Recently, it was found that the use of $\mathrm{COC}$ increases plasma renin values by $600 \%$, which may explain why the use of this drug is a risk factor for the development of systemic arterial hypertension. Although the use of Injectable Hormonal Contraceptives (IHC) is increasing, we have not found clinical studies that addressed the topic, demonstrating a gap in the scientific literature. OBJECTIVE: Compare the values of plasma renin, angiotensin-converting enzyme 1 and aldosterone of women using IHC with women who do not use any hormone-based contraceptives. METHODS: Protocol of a comparative observational cross-sectional study, composed of women aged between 18 and 30 years, eutrophic, irregularly active by the International Physical Activity Questionnaire, short version, who have been in continuous use of IHC for at least 6 months or that do not use. The sample will be for convenience and the selected participants will sign the informed consent form. Subsequently, they will answer a standard questionnaire, undergo a physical examination, and be sent to collect blood samples.

KEYWORDS: Contraceptive Agents. Renin-Angiotensin System. Hypertension. Women's Health 


\section{Introdução}

As Nações Unidas reportaram que em 2019 o número de mulheres em idade reprodutiva fazendo uso de métodos contraceptivos foi superior a 920 milhões. ${ }^{1}$ Dentre as possibilidades ofertadas para o controle de natalidade existem os contraceptivos orais, a forma mais utilizada de contracepção, chegando a $200 \mathrm{mi}-$ Ihões de usuárias desde sua iniciação pela medicina na década de $1960 .{ }^{1}$

Desde 2013, nosso grupo de pesquisa tem apresentado resultados que sugerem fortemente que mulheres sem outros fatores de risco, em uso de Contraceptivos Orais Combinados (COC), apresentam maior valor de proteína $C$ reativa ${ }^{2}$, de lipemia pós-prandial ${ }^{3}$, de lipoproteína de baixa densidade oxidada ${ }^{4}$, além de diminuição da sensibilidade insulínica ${ }^{5}$, quando comparadas a suas congêneres sem uso de COC. No entanto, o uso de Contraceptivo Hormonal Injetável (CHI) tem aumentado. No Brasil, uma estimativa feita em 2014, apontou que 5\% das mulheres residentes em área urbana, com idade de 15 a 49 anos, não gestantes e que fazem uso de algum contraceptivo, utilizam $\mathrm{CHI} .{ }^{6}$

Recentemente, observamos que o uso de COC eleva os valores de renina plasmática em $600 \%$, o que pode ser uma explicação do porque o uso desse fármaco é um fator de risco para o desenvolvimento de hipertensão arterial sistêmica, como apontou uma metanálise incluindo 24 estudos e mais de 250.000 participantes. ${ }^{8}$ A renina é parte fundamental do Sistema Renina-Angiotensina (SRA), sistema que atualmente tem sido discutido na ciência mundial de forma ingente, já que a SARS-CoV-2 utiliza a enzima conversora de angiotensina 2, outro elemento desse sistema, para penetrar as células do corpo humano. 9

A despeito da interação entre o SRA e a SARS-CoV-2, sabemos que esse sistema regula uma série de variáveis fisiológicas que impactam diretamente em condições clínicas, como na pressão arterial sistêmica ${ }^{10}$, na massa e força muscular miocárdica. ${ }^{11} \mathrm{~A}$ utilização crescente de $\mathrm{CHI}$ e a importância clínica do SRA, nos estimulou a investigar os efeitos dos $\mathrm{CHI}$ sobre o SRA. Ademais, numa busca realizada na base de dados Pubmed, não encontramos estudos clínicos que tivessem estudado esse tema, identificando uma lacuna ainda existente na literatura científica.
Comparar os valores de renina plasmática, enzima conversora de angiotensina 1 e aldosterona de muIheres que utilizam $\mathrm{CHI}$ com mulheres que não utilizam nenhum contraceptivo à base de hormônio.

\section{Métodos}

\section{Desenho de estudo}

A pesquisa se caracteriza como um protocolo de estudo observacional comparativo de corte transversal.

\section{Contexto}

A amostra será integrada por usuárias do Programa de Planejamento Familiar do Ministério da Saúde no município de Ibicaraí - BA, recrutadas nas Unidades de Saúde da Família do município e estratificadas em dois grupos: grupo contraceptivo injetável (GCI), composto por voluntárias que fazem uso de $\mathrm{CHI}$ há pelo menos 6 meses, e o grupo sem contraceptivo (GSI) formado por voluntárias que não fazem uso de qualquer $\mathrm{CHI}$ há pelo menos 6 meses. O período previsto para o recrutamento das voluntárias, coleta de dados, realização de exame físico e coleta laboratorial é no primeiro semestre de 2021.

\section{População do estudo}

Mulheres jovens irregularmente ativas que estão em uso continuado de $\mathrm{CHI}$ há pelo menos 6 meses e por mulheres com as mesmas condições clínicas que não fazem uso de $\mathrm{CHI}$.

\section{Critérios de elegibilidade}

Serão inclusas mulheres com idade entre 18 e 30 anos, eutróficas, irregularmente ativas pelo Questionário Internacional de Atividade Física versão curta ${ }^{12}$, que estão em uso continuado de $\mathrm{CHI}$ há pelo menos 6 meses e mulheres com as mesmas condições clínicas que não fazem uso de $\mathrm{CHI}$ há pelo menos 6 meses.

Serão exclusas mulheres com dados clínicos inconsistentes, diabéticas, hipertensas ou em uso de anti-hipertensivo, com doenças cardíacas, renais ou 
metabólicas, tabagistas ou etilistas avaliadas pelo questionário Cut-down, Annoyed, Guilty e Eye-opener (CAGE). Além disso, serão excluídas mulheres que apresentem síndrome do ovário policístico pois esta condição gera complicações de desregulação do ciclo menstrual, câncer de endométrio e distúrbios metabólicos, como a disfunção da célula beta-pancreática e elevação de marcadores inflamatórios, tendo como exemplo a Proteína $\mathrm{C}$ reativa (PCR). $\underline{\underline{13}}$

\section{Coleta de dados}

As voluntárias selecionadas responderão a um questionário padrão e serão submetidas a um exame físico, ambos com a função de coletar informações gerais sobre as características da amostra. O exame físico será composto por medidas de Frequência Cardíaca (FC) e Pressão Arterial (PA) em repouso, massa corporal total, estatura e posteriormente cálculo do Índice de Massa Corpórea (IMC) e Circunferência Abdominal (CA). Essa coleta de dados ocorrerá no espaço físico das salas de triagem das Unidades de Saúde da Família do município.

Para mensuração da FC será utilizado um cardiofrequencímetro de pulso da marca Choicemmed. Já para a mensuração da PA, será seguido as recomendações da American Heart Association utilizado um esfigmomanômetro para os adultos devidamente calibrado pelo Instituto Nacional de Metrologia (INMETRO) e um estetoscópio duo-sonic, ambos da marca Premyum.

A estatura será medida com o auxílio de um estadiômetro profissional Sanny com precisão de $0,1 \mathrm{~cm}$, executada com os sujeitos descalços e com os glúteos e ombros apoiados em um encosto vertical. A massa corporal total será mensurada com balança digital Filizola, capacidade máxima de 150kg, aferida pelo INMETRO e com certificado próprio especificando margem de erro de $\pm 100 \mathrm{~g}$.

O IMC será calculado com as medidas de massa e altura, de acordo com a seguinte fórmula: IMC = massa (kg) / altura2 (cm). Os pontos de corte de IMC adotados serão os preconizados pela IV Diretriz Brasileira sobre Dislipidemias e Prevenção da Aterosclerose do Departamento de Aterosclerose da Sociedade Brasileira de Cardiologia ${ }^{14}$, sendo baixo peso (IMC <
18,5); eutrofia (IMC 18,5-24,9); sobrepeso (IMC 2529,9) e obesidade (IMC $\geq 30$ ).

Para medida da circunferência abdominal (CA), será utilizada fita métrica metálica e flexível, marca Wiso, com definição de medida de $0,1 \mathrm{~cm}$. A CA será mensurada na menor curvatura localizada entre as costelas e a crista ilíaca sem comprimir os tecidos. Quando não for possível identificar a menor curvatura, será obtido a medida dois centímetros acima da cicatriz umbilical. Os pontos de corte adotados para CA serão estipulados de acordo com o grau de risco para doenças cardiovasculares, sendo, para mulheres (CA $\geq 88 \mathrm{~cm}$ ) e para homens $(C A \geq 102 \mathrm{~cm}) \cdot \frac{13}{}$

\section{Coleta laboratorial}

Todas as voluntárias serão orientadas a realizarem jejum de 12 horas, a não alterarem sua dieta na semana do teste, a não praticarem nenhum esforço físico diferente do habitual e a não ingerir bebidas alcoólicas 24 horas antes do exame laboratorial; e serão encaminhadas para o Laboratório de Análises Clínicas no município de Ibicaraí - BA para coleta das amostras sanguíneas.

As coletas serão realizadas em dois momentos, no tempo 0 e após 60 minutos de repouso. No tempo 0 serão coletados $10 \mathrm{~mL}$ de sangue para dosagem de transaminase glutâmica oxalacética (TGO), transaminase glutâmica pirúvica (TGP) para avaliação da função hepática, e glicemia para avaliação dos níveis de açúcar. Os valores para TGO e TGP serão obtidos pelo método colorimétrico (Reitman- Frankel) e os valores para glicemia de jejum serão obtidos pelo método colorimétrico enzimático de Trinder.

Posteriormente, é necessário que as voluntárias permaneçam em repouso na posição decúbito dorsal por 60 minutos para dosagem de renina plasmática, enzima conversora de angiotensina I e aldosterona. $\cdot \frac{15}{5}$ A renina será medida por método de radioimunoensaio cinético no plasma com EDTA, os valores para enzima conversora de angiotensina serão obtidos pelo método cinético optimizado U.V e aldosterona pelo método radioimunoensaio. A organização das coletas pode ser observada na figura 1 . 


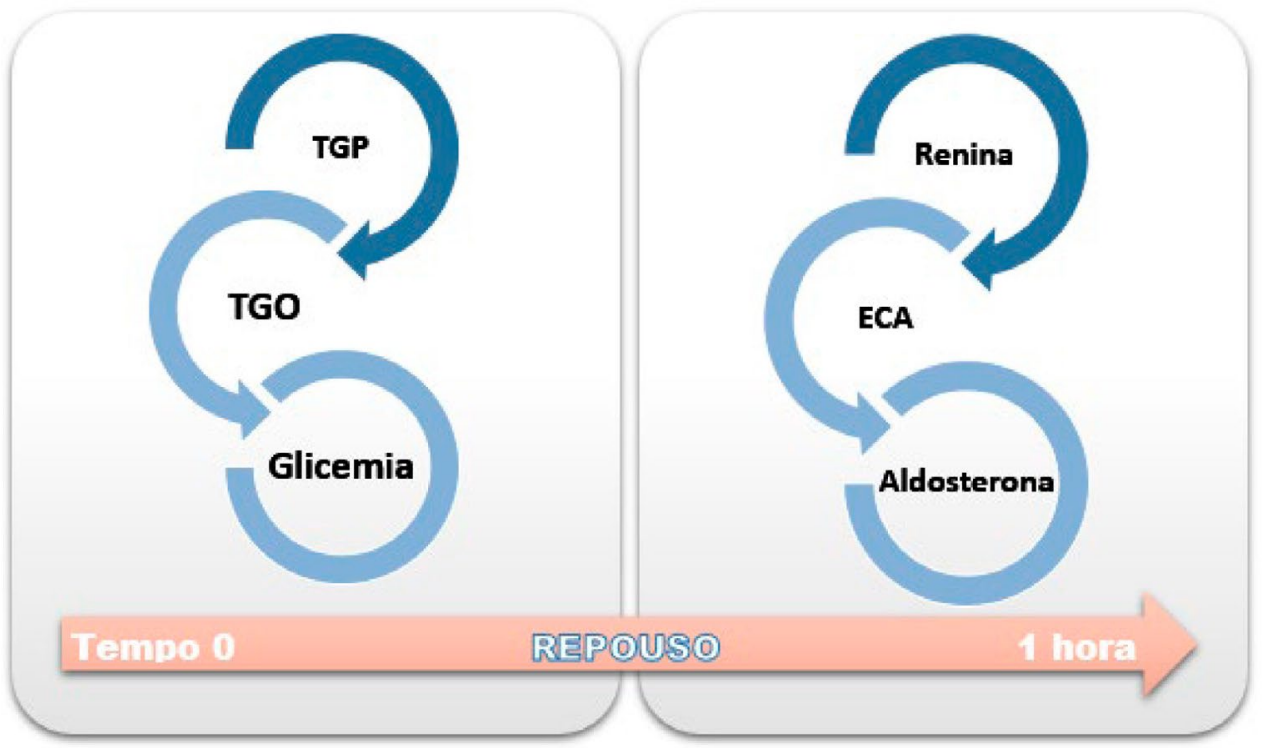

ECA: Enzima Conversora de Angiotensina; TGO: Transaminase Glutâmica Oxalacética; TGP: Transaminase Glutâmica Pirúvica.

\section{Aspectos éticos}

Este estudo foi aprovado pelo Comitê de Ética em Pesquisa da Escola Bahiana de Medicina e Saúde Pública, sob CAAE $n^{\circ}$ 35292220.2.0000.5544. As voluntárias selecionadas serão orientadas previamente quanto à realização da pesquisa e assinarão o termo de consentimento livre e esclarecido. Serão preenchidas duas vias, uma para posse da voluntária e outra para posse do pesquisador. Conforme a Resolução n 466/2012, do Conselho Nacional de Saúde, serão respeitados todos os preceitos éticos e legais de pesquisa com seres humanos. A Secretaria Municipal de Saúde do munícipio de Ibicaraí - Ba e o Laboratório de Análises Clínicas receberam e assinaram uma carta de anuência, constando as informações do estudo e a liberação para aplicação da pesquisa nas Unidades de Saúde da família, bem como coleta sanguínea.

\section{Variáveis}

Variável preditora - uso de $\mathrm{CHI}$

Variáveis de desfecho - Renina plasmática, enzima conversora de angiotensina 1 e aldosterona.

\section{Desfechos primários}

Renina plasmática, enzima conversora de angiotensina 1 e aldosterona.

\section{Tamanho da amostra}

A amostra será por conveniência objetivando 60 participantes.

\section{Análise Estatística}

Inicialmente, para verificar a distribuição dos dados serão aplicados testes de simetria e curtose e o teste de Shapiro-Wilk. Os valores das variáveis com comportamento normal serão descritos em média e desvio padrão e os valores das variáveis não paramétricas em mediana e intervalo interquartil. As variáveis categóricas serão apresentadas como frequência absoluta e relativa. Para a comparação intergrupos das variáveis paramétricas será utilizado o teste t de Student não pareado bidirecional e para as variáveis não paramétricas o teste de Mann-Whitney. 
O nível de significância adotado para este estudo será de $5 \%$ e todos os dados serão analisados utilizando o software estatístico Statistical Package for the Social Sciences (SPSS) versão 22.0.

\section{Contribuições dos autores}

Santos PA participou da concepção e redação do manuscrito. Oliveira AM participou da redação do manuscrito. Petto J participou da concepção, redação do manuscrito e revisão crítica do manuscrito quanto ao conteúdo intelectual.

\section{Conflitos de interesses}

Nenhum conflito financeiro, legal ou político envolvendo terceiros (governo, empresas e fundações privadas, etc.) foi declarado para nenhum aspecto do trabalho submetido (incluindo, mas não se limitando a subvenções e financiamentos, participação em conselho consultivo, desenho de estudo, preparação de manuscrito, análise estatística, etc.).

\section{Referências}

1. United Nations, Department of Economic and Social Affairs, Population Division (2019). Contraceptive Use by Method 2019: Data Booklet [Internet]. Disponível em: https:// www.un.org/development/desa/pd/sites/www.un.org. development.desa.pd/files/files/documents/2020/Jan/un_2019. contraceptiveusebymethod databooklet.pdf

2. Petto J, Pereira LS, Santos ACN, Giesta BA, Melo TA, Ladeia AMT. Inflamação Subclínica em Mulheres que Utilizam Contraceptivo Oral. Rev Bras Cardiol [Internet]. 2013;26(6):465-71. Disponível em: http://www.onlineijcs.org/english/sumario/26/pdf/v26n6a08. pdf

3. Petto J, Vasques LMR, Pinheiro RL, Giesta BA, Santos ACN, Gomes Neto M, et al. Comparação da Lipemia Pós-Prandial de Mulheres que Utilizam e Não Utilizam Contraceptivo Oral. Arq. Bras. Cardiol. 2014;103(3):245-50. https://doi.org/10.5935/ abc. 20140080

4. Santos ACN, Petto J, Diogo DP, Seixas CR, Souza LH, Araújo WS, et al. Elevação da Lipoproteína de Baixa Densidade Oxidada em Usuárias de Contraceptivo Oral Combinado. Arq. Bras. Cardiol. 2018;111(6):764-70. https://doi.org/10.5935/abc.20180194
5. Seixas CR, Petto J, Sacramento MS, Santos ACN, Wagmacker DS, Ladeia AMT. Is the use of combined oral contraceptive able to change the insulin sensitivity? Int J Curr Res [Internet]. 2019;11(7):5793-8. Disponível em: http://journalcra.com/article/ use-combined-oral-contraceptive-able-change-insulin-sensitivity

6. Farias MR, Leite SN, Tavares NUL, Oliveira MA, Arrais PSD, Bertoldi $A D$, et al. Utilização e acesso a contraceptivos orais e injetáveis no Brasil. Rev Saude Publica. 2016;50(supl 2):14s. https://doi.org/10.1590/S1518-8787.2016050006176

7. Oliveira AA, Petto J, Diogo DP, Santos ACN, Sacramento MS, Ladeia AMT. Plasma Renin in Women Using and Not Using Combined Oral Contraceptive. Int J Cardiovasc Sci. 2020;33(3):20814. https://doi.org/10.36660/ijcs.20180021

8. Liu H, Yao J, Wang W, Zhang D. Association between duration of oral contraceptive use and risk of hypertension: A meta-analysis. J Clin Hypertens (Greenwich). 2017;19(10):1032-41. https://doi. org/10.1111/jch.13042

9. Vaduganathan M, Vardeny O, Michel T, McMurray JJV, Pfeffer MA, Solomon SD. Renin- angiotensin-aldosterone system inhibitors in patients with Covid-19. N Engl J Med 2020;382(17):1653-9. https://doi.org/10.1056/nejmsr2005760

10. Campbell DJ. The renin-angiotensin and the kallikrein-kinin system. Int J Biochem Cell Biol. 2003;35(6):784-91. https://doi. org/10.1016/S1357-2725(02)00262-5

11. Dzau VJ. Circulating versus local renin-angiotensin system in cardiovascular homeostasis. Circulation. 1988;77(6 Pt 2):14-13. Citado em: PMID: 3286045

12. Matsudo S, Araújo T, Matsudo V, Andrade D, Andrade $E$, Oliveira LC, et al. Questionário internacional de atividade física (IPAQ): estudo de validade e reprodutibilidade no Brasil. Atividade Física \& Saúde. 2001;6(2):5-18. https://doi.org/10.12820/ rbafs.v.6n2p5-18

13. Khalsa JH, Genser S, Coates P, Francis H. Interventions for metabolic and endocrine complications of human immunodeficiency virus/acquired immune deficiency syndrome and illicit drug use. Clin Infect Dis. 2003;37(supl 2):S37-42. https:// doi.org/10.1086/375890

14. Xavier HT, Izar MC, Faria Neto JR, Assad MH, Rocha VZ, Sposito AC, et al. V Diretriz Brasileira Sobre Dislipidemias e Prevenção da Aterosclerose. Arq Bras Cardiol. 2013;101(4 supl 1). https://doi. org/10.5935/abc.20135010

15. Hermes Pardini - Medicina, Saúde e Bem Estar. Manual de Exames: Hermes Pardini; 2015/2016. 